\title{
On the interaction of different types of ligands binding to the same molecule Part II: systems with $\mathbf{n}$ to 2 and $\mathbf{n}$ to 3 binding sites
}

\author{
Johannes W. R. Martini • Martin Schlather • \\ G. Matthias Ullmann
}

Received: 7 October 2012 / Accepted: 18 October 2012 / Published online: 11 November 2012 C) The Author(s) 2012. This article is published with open access at Springerlink.com

\begin{abstract}
In the first part of this work we formulated the decoupled sites representation for two different types of ligands and highlighted special properties of the case of $n$ binding sites for ligand $L_{1}$ and one binding site for ligand $L_{2}$. Moreover, for this case, we identified the microstate constants as unique components all decoupled molecules share. In the second part on hand, we investigate the cases with $(n, 2)$ and $(n, 3)$ binding sites. As it is difficult to solve the system of equations occurring when a molecule with more than one binding site for both ligands shall be decoupled, we present applicable calculation methods which exploit the special structure of the system of equations. Moreover, we investigate which unique properties all decoupled molecules share and show that for two different decoupled molecules with the same binding polynomial, not all microstate constants of a certain macrostate are permutations of the microstate constants of the other molecule.
\end{abstract}

Keywords Decoupled sites representation - Protonation · Binding polynomial $\cdot$ Interaction energy $\cdot$ Binding energy $\cdot$ Ligand binding $\cdot$ Electron transfer $\cdot$ Photosynthesis $\cdot$ Receptor

J. W. R. Martini (凶)

Institut für Mathematische Stochastik, Georg-August Universität Göttingen, Göttingen, Germany e-mail: jmartin2@uni-goettingen.de

M. Schlather

Institut für Mathematik, Universität Mannheim, Mannheim, Germany

e-mail: schlather@math.uni-mannheim.de

G. M. Ullmann

Bioinformatik/Strukturbiologie, Universität Bayreuth, Bayreuth, Germany

e-mail: Matthias.Ullmann@uni-bayreuth.de 


\section{Introduction}

We regard a molecule $M$ in solution to which another molecule $L$ can bind reversibly at several binding sites. If the activity (or concentration) of $L$ is changed, the average amount of ligand $L$ bound to the molecule $M$ in equilibrium will change, too. In this work the general objects of interest are titration curves as functions in the ligand activities which describe the average binding of two different ligands to a molecule as a whole (overall titration) or to a certain site, in equilibrium. We give a short summary to recall the basics. A more detailed description is given in the first part of this work [7].

In the underlying model, the equilibrium binding properties of the molecule $M$ are described by energies of microstates which are mapped to rational functions in the ligand activities (the titration curves). With $n=n_{1}+n_{2}$ denoting the number of binding sites for both ligands $L_{i}$, a microstate $k$ is an $n$-tuple illustrating the binding state of an individual molecule:

$$
k=\left(x_{1}^{k}, \ldots, x_{n}^{k}\right) \quad x_{i}^{k} \in\{0,1\} \forall i \in\{1, \ldots, n\}
$$

and

$$
x_{i}^{k}=1 \Longleftrightarrow \text { in microstate } k \text {, a ligand is bound to site } i \text {. }
$$

$K$ denotes the set of all microstates $k$. We use a simplified model in which the microstate energies are sums of binding energies (energy difference if a certain site is (un)occupied) and pairwise interaction energies describing whether an occupation of a certain site influences the binding probability of a ligand to another site. This model is simplified as, in an extended model, additional summands of interaction energy resulting from interaction of three or more binding sites would be incorporated. Here, in this simplified model, we profit from a relieved notation and the main results carry over to the more general setup. Thus, the binding properties of ligands $L_{1}$ and $L_{2}$ to the molecule $M$ can be characterized by an $\frac{n(n+1)}{2}$-tuple of binding $\left(g_{i}\right)$ and interaction constants $\left(w_{i, j}\right)$

$$
M=\left(g_{1}^{M}, \ldots, g_{n}^{M}, w_{1,2}^{M}, \ldots, w_{n-1, n}^{M}\right) \in \mathbb{C}^{* m},
$$

where $\mathbb{C}^{*}=\mathbb{C} \backslash\{0\}$. Its entries are called "binding constants" and "interaction constants", which are given by

$$
g_{i}^{M}:=e^{-\beta G_{i}^{M}}
$$

and

$$
w_{i, j}^{M}:=e^{-\beta W_{i, j}^{M}}
$$

where $G_{i}^{M}$ denotes the binding energy of site $i, W_{i, j}^{M}$ the interaction energy of sites $i$ and $j$, and $\beta$ a constant depending on the temperature (which is assumed constant). 
The constant $g(k)$ of a microstate $k$ is given by

$$
g(k):=\left(\prod_{i=1}^{n}\left(g_{i}^{x_{i}^{k}} \prod_{j=i+1}^{n} w_{i, j}^{x_{i}^{k} x_{j}^{k}}\right)\right)
$$

in the model with only pairwise interaction. The binding polynomial in the ligand activities $\Lambda$ and $\kappa$ of a molecule $M$ with $n_{1}$ binding sites for ligand $L_{1}$ and $n_{2}$ binding sites for ligand $L_{2}$, which is the denominator of all titration curves, writes

$$
P_{M}(\Lambda, \kappa)=\sum_{k \in K} g(k) \Lambda^{l_{1}(k)} \kappa^{l_{2}(k)}
$$

with $l_{1}(k):=\sum_{i=1}^{n_{1}} x_{i}^{k}$ and $l_{2}(k):=\sum_{i=n_{1}+1}^{n_{1}+n_{2}} x_{i}^{k}$ denoting the number of bound ligands of both types and $g(k)$ again the microstate constant of state $k$. The average amount of bound ligand to site $r$ in equilibrium is given by

$$
\left\langle x_{r}\right\rangle=\frac{\sum_{\left\{k \in K \mid x_{r}^{k}=1\right\}} g(k) \Lambda^{l_{1}(k)} \kappa^{l_{2}(k)}}{\sum_{k \in K} g(k) \Lambda^{l_{1}(k)} \kappa^{l_{2}(k)}}=: \frac{Z_{M}^{r}(\Lambda, \kappa)}{P_{M}(\Lambda, \kappa)} .
$$

With $1, \ldots, n_{1}$ denoting the binding sites for ligand $L_{1}$ and $A_{1}, \ldots, A_{n_{2}}$ those for $L_{2}$, Eq. (4) leads to the following overall titration curves for ligands $L_{1}$ and $L_{2}$ :

$$
\begin{aligned}
\left\langle X_{1}\right\rangle & =\frac{\sum_{r=1}^{n_{1}} Z_{M}^{r}(\Lambda, \kappa)}{P_{M}(\Lambda, \kappa)} \\
\left\langle X_{2}\right\rangle & =\frac{\sum_{r=A_{1}}^{A_{n_{2}}} Z_{M}^{r}(\Lambda, \kappa)}{P_{M}(\Lambda, \kappa)}
\end{aligned}
$$

The situation with only one type of ligand $L_{1}\left(n_{2}=0\right)$ has been investigated for a long time $[1-6,12]$ and it is well described. An important feature within the theory of ligand binding if only one ligand is present is the decoupled sites representation (DSR) which states that for any overall titration curve, a hypothetical molecule with non-interacting binding sites exhibiting this overall titration behavior exists [8-10]. In the first part of this work, we formulated the DSR for molecules with two different types of ligands as conjecture and highlighted the case of $n_{1}$ to one binding sites for the two different ligands [7]. In this part we will treat the cases $(n, 2)$ and $(n, 3)$ exemplarily to generate an intuition for the situation of $\left(n_{1}, n_{2}\right)$ ligand binding sites, for which certain statements are difficult to prove. Moreover, we present numerical methods for the calculation of corresponding decoupled systems.

\section{$2 \mathrm{n}$ to two binding sites}

We prove the DSR for the case of two binding sites for both ligands and present an iterative approach to calculate decoupled systems with $(n, 2)$ bindings sites. Exemplarily, this is used subsequently to decouple a molecule with $(4,2)$ binding sites. 
2.1 Two to two binding sites

We formulate the DSR as proposition for the case $n_{1}=n_{2}=2$.

Proposition 1 Let

$$
M=\left(g_{1}^{M}, g_{2}^{M}, g_{A}^{M}, g_{B}^{M}, w_{1,2}^{M}, w_{1, A}^{M}, w_{1, B}^{M}, w_{2, A}^{M}, w_{2, B}^{M}, w_{A, B}^{M}\right)
$$

be a molecule with two binding sites for each type of ligand. Then a molecule

$$
N=\left(g_{1}, g_{2}, g_{A}, g_{B}, 1, w_{1, A}, w_{1, B}, w_{2, A}, w_{2, B}, 1\right)
$$

exists, with

$$
P_{M}=P_{N}
$$

Proof We will use the special structure of the algebraic equations we are dealing with to prove Proposition 1. Let

$$
M=\left(g_{1}^{M}, g_{2}^{M}, g_{A}^{M}, g_{B}^{M}, w_{1,2}^{M}, w_{1, A}^{M}, w_{1, B}^{M}, w_{2, A}^{M}, w_{2, B}^{M}, w_{A, B}^{M}\right)
$$

be a molecule with bp

$$
\begin{aligned}
P_{M}= & a_{2,2} \Lambda^{2} \kappa^{2}+a_{2,1} \Lambda^{2} \kappa+a_{2,0} \Lambda^{2} \\
& +a_{1,2} \Lambda \kappa^{2}+a_{1,1} \Lambda \kappa+a_{1,0} \Lambda+a_{0,1} \kappa^{2}+a_{0,1} \kappa+1 .
\end{aligned}
$$

We seek for a molecule $N=\left(g_{1}, g_{2}, g_{A}, g_{B}, 1, w_{1, A}, w_{1, B}, w_{2, A}, w_{2, B}, 1\right)$ with the same binding polynomial. The bp gives a system of eight equations corresponding to its coefficients

$$
\begin{aligned}
& a_{2,2}=g_{1} g_{2} g_{A} g_{B} w_{1, A} w_{1, B} w_{2, A} w_{2, B} \\
& a_{2,1}=g_{1} g_{2} g_{A} w_{1, A} w_{2, A}+g_{1} g_{2} g_{B} w_{1, B} w_{2, B} \\
& a_{2,0}=g_{1} g_{2} \\
& a_{1,2}=g_{1} g_{A} g_{B} w_{1, A} w_{1, B}+g_{2} g_{A} g_{B} w_{2, A} w_{2, B} \\
& a_{1,1}=g_{1} g_{A} w_{1, A}+g_{1} g_{B} w_{1, B}+g_{2} g_{A} w_{2, A}+g_{2} g_{B} w_{2, B} \\
& a_{1,0}=g_{1}+g_{2} \\
& a_{0,2}=g_{A} g_{B} \\
& a_{0,1}=g_{A}+g_{B}
\end{aligned}
$$

The binding energies $g_{i}$ can be calculated using the equations given by the coefficients with only one type of ligand. According to Vieta's formulas (for more details see Corollary 1, [7]):

$$
\left(g_{1}, g_{2}\right)=\left(-\frac{1}{\Lambda_{z_{1}}},-\frac{1}{\Lambda_{z_{2}}}\right)
$$


with $\Lambda_{z_{i}}$ denoting the roots of

$$
a_{2,0} \Lambda^{2}+a_{1,0} \Lambda+1
$$

Analogously, $\left(g_{A}, g_{B}\right)$ can be calculated using $a_{0,2}, a_{0,1}$. Thus, in general for any choice of $\left(n_{1}, n_{2}\right)$, the subsystem of equations given by the coefficients $a_{i, 0}$ and $a_{0, j}$,

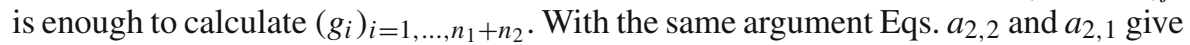
the products $g_{A} w_{1, A} w_{2, A}$ and $g_{B} w_{1, B} w_{2, B}$ by calculating the roots of

$$
\frac{a_{2,2}}{g_{1} g_{2}} \Lambda^{2}+\frac{a_{2,1}}{g_{1} g_{2}} \Lambda+1
$$

Analogously, $a_{2,2}$ and $a_{1,2}$ give $g_{1} w_{1, A} w_{1, B}$ and $g_{2} w_{2, A} w_{2, B}$. This means we have already found $\left(g_{i}\right)$ solving the subsystem $\left\{a_{0, j}, a_{j, 0}\right\}$, and products $\left(g_{i} w_{i, A} w_{i, B}\right)_{i=1,2}$, $\left(g_{j} w_{1, j} w_{2, j}\right)_{j=A, B}$ such that all equations, except for $a_{1,1}$ are solved. The remaining question is whether the products can be factorized such that all required conditions are fulfilled. As we know the binding constants we can rewrite the conditions on the products to

$$
\begin{gathered}
w_{1, A} w_{1, B}=b_{1} \\
w_{2, A} w_{2, B}=b_{2} \\
w_{1, A} w_{2, A}=b_{A} \\
w_{1, B} w_{2, B}=b_{B} \\
g_{1} g_{A} w_{1, A}+g_{2} g_{B} w_{2, B}+g_{1} g_{B} w_{1, B}+g_{2} g_{A} w_{2, A}=a_{1,1}
\end{gathered}
$$

If there exist $\left(w_{i, j}\right)$ solving system (9) then the whole system (7) will have a solution. Rearranging the first four equations of system (9) shows that we can solve them simultaneously for any choice of $w_{1, A}$ if and only if $\frac{b_{A} b_{B}}{b_{2} b_{1}}=1$. However, this is true as the $b_{i} \mathrm{~s}$ are derived from the roots of polynomials and fulfill in particular

$$
a_{2,2}=w_{1, A} w_{1, B} w_{2, A} w_{2, B} \prod_{i=1}^{B} g_{i}=b_{1} b_{2} \prod_{i=1}^{B} g_{i}=b_{A} b_{B} \prod_{i=1}^{B} g_{i}
$$

Consequently, we can solve the first four equations to receive expressions depending on $w_{1, A}$, only, and plug them into the last equation which gives a polynomial of degree two with at least one root non-zero.

A generalization of this procedure might be adequate to prove the DSR without fixing $n_{1}$ and $n_{2}$.

2.2 Decoupling a molecule with four and two binding sites for different ligands

Here, we illustrate the decoupling of a hypothetical molecule with four binding sites for electrons (ligand $L_{1}$ ) and two binding sites for protons (ligand $L_{2}$ ). Let the sites 
for electrons be denoted by $1, \ldots, 4$ and the proton binding sites be called $A, B$. Even though the hypothetical molecule has only six binding sites decoupling is challenging. To find a decoupled molecule for a system with four and two binding sites we have to solve system (10) (below) consisting of 14 polynomial equations (one equation per coefficient) with 14 variables given by the binding constants and the interaction constants. To facilitate identifying the structure of the system, which is required to understand how we find solutions, we use the following substitutions:

$$
\begin{aligned}
\xi_{i}:= & g_{i} w_{i, A} w_{i, B} \\
a_{4,1}^{j}:= & g_{1} g_{2} g_{3} g_{4} g_{j} w_{1, j} w_{2, j} w_{3, j} w_{4, j} \\
a_{3,1}^{j}:= & g_{1} g_{2} g_{3} g_{j} w_{1, j} w_{2, j} w_{3, j}+g_{1} g_{2} g_{4} g_{j} w_{1, j} w_{2, j} w_{4, j} \\
& +g_{1} g_{3} g_{4} g_{j} w_{1, j} w_{3, j} w_{4, j}+g_{2} g_{3} g_{4} g_{j} w_{2, j} w_{3, j} w_{4, j} \\
a_{2,1}^{j}:= & g_{1} g_{2} g_{j} w_{1, j} w_{2, j}+g_{1} g_{3} g_{j} w_{1, j} w_{3, j}+g_{1} g_{4} g_{j} w_{1, j} w_{4, j} \\
& +g_{2} g_{3} g_{j} w_{2, j} w_{3, j}+g_{2} g_{4} g_{j} w_{2, j} w_{4, j}+g_{3} g_{4} g_{j} w_{3, j} w_{4, j} \\
a_{1,1}^{j}= & g_{1} g_{j} w_{1, j}+g_{2} g_{j} w_{2, j}+g_{3} g_{j} w_{3, j}+g_{4} g_{j} w_{4, j}
\end{aligned}
$$

with $i \in\{1,2,3,4\}$ and $j \in\{A, B\}$. A look at system (10) reveals that it consists of three systems of the type described in Corollary 1 of [7] and a system which is a sum of two systems of the same structure $\left(\left\{a_{i, 1}\right\}_{i=1,2,3,4}\right)$.

$$
\begin{aligned}
\frac{a_{4,2}}{g_{A} g_{B}} & =\xi_{1} \xi_{2} \xi_{3} \xi_{4} \\
\frac{a_{3,2}}{g_{A} g_{B}} & =\xi_{1} \xi_{2} \xi_{3}+\xi_{1} \xi_{2} \xi_{4}+\xi_{1} \xi_{3} \xi_{4}+\xi_{2} \xi_{3} \xi_{4} \\
\frac{a_{2,2}}{g_{A} g_{B}} & =\xi_{1} \xi_{2}+\xi_{1} \xi_{3}+\xi_{1} \xi_{4}+\xi_{2} \xi_{3}+\xi_{2} \xi_{4}+\xi_{3} \xi_{4} \\
\frac{a_{1,2}}{g_{A} g_{B}} & =\xi_{1}+\xi_{2}+\xi_{3}+\xi_{4} \\
a_{4,1} & =a_{4,1}^{A}+a_{4,1}^{B} \\
a_{3,1} & =a_{3,1}^{A}+a_{3,1}^{B} \\
a_{2,1} & =a_{2,1}^{A}+a_{2,1}^{B} \\
a_{1,1} & =a_{1,1}^{A}+a_{1,1}^{B} \\
a_{0,2} & =g_{A} g_{B} \\
a_{0,1} & =g_{A}+g_{B} \\
a_{4,0} & =g_{1} g_{2} g_{3} g_{4} \\
a_{3,0} & =g_{1} g_{2} g_{3}+g_{1} g_{2} g_{4}+g_{1} g_{3} g_{4}+g_{2} g_{3} g_{4}
\end{aligned}
$$




$$
\begin{aligned}
& a_{2,0}=g_{1} g_{2}+g_{1} g_{3}+g_{1} g_{4}+g_{2} g_{3}+g_{2} g_{4}+g_{3} g_{4} \\
& a_{1,0}=g_{1}+g_{2}+g_{3}+g_{4}
\end{aligned}
$$

To find a decoupled system for Example 1, we tried to use the standard command "algsys" of the computer algebra system Maxima to solve the system. However, it was too complicated to be solved directly by this general approach. Instead we used the special structure of the system to deduce an iterative procedure with decoupled systems as fixed point: Regarding the system of polynomial equations we see that the equations given by $a_{4,0}, a_{3,0}, a_{2,0}, a_{1,0}$ do neither contain any interaction constant as variable, nor the binding constants $g_{A}, g_{B}$. Thus, we have four equations with the four variables $g_{1}, g_{2}, g_{3}, g_{4}$. This subsystem can be solved using the well known procedure: $g_{i}=-\frac{1}{z_{i}}$ where $z_{i}$ denote the roots of the polynomial

$$
P_{1}(x)=a_{4,0} x^{4}+a_{3,0} x^{3}+a_{2,0} x^{2}+a_{1,0} x+1 .
$$

Analogously, coefficients $a_{0,2}, a_{0,1}$ immediately give a solution for $\left(g_{A}, g_{B}\right)$. Hence, the binding energies are unique, except for permutations. We chose any permutation which means we fix the binding constants. The products $\xi_{i}=g_{i} w_{i, A} w_{i, B}$ can be calculated using equations $a_{4,2}, a_{3,2}, a_{2,2}, a_{1,2}$ : Again, these products are the negative inverses of the roots of the polynomial

$$
P_{2}(x)=\frac{a_{4,2}}{g_{A} g_{B}} x^{4}+\frac{a_{3,2}}{g_{A} g_{B}} x^{3}+\frac{a_{2,2}}{g_{A} g_{B}} x^{2}+\frac{a_{1,2}}{g_{A} g_{B}} x+1 .
$$

Note, that this is the major step which distinguishes between the different decoupled molecules: We have fixed an order of the binding constants previously, and have to relate the roots of $P_{2}$ and the products $g_{i} w_{i, A} w_{i, B}$. In general, we will receive different decoupled molecules for different permutations of the roots of $P_{2}$ (if they do not coincide due to identical binding constants, etc.). As we know the binding constants $g_{i}$, these solutions give conditions on $w_{i, A} w_{i, B}$. Regarding equations $a_{4,1}, a_{3,1}, a_{2,1}, a_{1,1}$ we see that the system is the sum of two "ordinary" systems which could be solved by the well known procedure previously described, if we knew $\left\{a_{i, 1}^{A}\right\}_{i=1,2,3,4}$. As this is not the case we use the iterative approach described in Proposition 2 .

Proposition 2 Let the algebraic system (10) be given. Let $\left(g_{i}\right)_{i=1, \ldots, 4}$ be a solution to the subsystem $\left\{a_{4,0}, a_{3,0}, a_{2,0}, a_{1,0}\right\},\left(g_{A}, g_{B}\right)$ be a solution to $\left\{a_{0,2}, a_{0,1}\right\}$. Furthermore, let the products $\left(w_{i, A} w_{i, B}\right)_{i=1, \ldots, 4}$ be a solution to $\left\{a_{4,2}, a_{3,2}, a_{2,2}, a_{1,2}\right\}$ for the given binding constants $\left(g_{i}\right)_{i=1,2,3,4, A, B}$ and let $\sigma^{n}$ be a sequence of permutations of $\{1, \ldots, 4\}$. We consider the following algorithm: 


$$
\begin{gathered}
a_{i, 1}^{A, 0}:=a_{i, 1}, \quad i \in\{1,2,3,4\} \\
P_{n}(x):=\frac{a_{4,1}^{A, n}}{g_{A}} x^{4}+\frac{a_{3,1}^{A, n}}{g_{A}} x^{3}+\frac{a_{2,1}^{A, n}}{g_{A}} x^{2}+\frac{a_{1,1}^{A, n}}{g_{A}} x+1 \\
\left(z_{1}^{n}, z_{2}^{n}, z_{3}^{n}, z_{4}^{n}\right):=\text { the roots } P_{n} \\
w_{i, A}^{n}:=-\frac{1}{z_{\sigma^{n}(i)}^{n} g_{i}} \\
w_{i, B}^{n}:=\frac{w_{i, A} w_{i, B}}{w_{i, A}^{n}}
\end{gathered}
$$

Calculate $a_{i, 1}^{B, n}$ using equations $\left\{a_{i, 1}\right\}_{i=1,2,3,4}$ and restart with

$$
a_{i, 1}^{A, n+1}:=a_{i, 1}-a_{i, 1}^{B, n}
$$

Then, $x=\left(w_{1, A}, w_{1, B}, w_{2, A}, w_{2, B}, w_{3, A}, w_{3, B}, w_{4, A}, w_{4, B}\right)$ is a solution to the subsystem $\left\{a_{i, n}\right\}_{i, n \neq 0}$ which satisfies the conditions on the products $\left(w_{i, A} w_{i, B}\right)_{i=1, \ldots, 4}$ if and only if there exists a permutation $\sigma \in \mathscr{S}_{4}$ such that $x$ is a fixed point of the algorithm with $\sigma^{n}=\sigma$.

Proof Let $x$ be a solution to the subsystem, satisfying the conditions on the products $\left(w_{i, A} w_{i, B}\right)_{i=1, \ldots, 4}$. Then

$$
w_{i, A}^{n}=w_{i, A} \Rightarrow w_{i, B}^{n}=\frac{w_{i, A} w_{i, B}}{w_{i, A}}=w_{i, B} \Rightarrow a_{i, 1}^{B, n}=a_{i, 1}^{B} \Rightarrow a_{i, 1}^{A, n+1}=a_{i, 1}^{A}
$$

which gives

$$
w_{i, A}^{n+1}=w_{i, A}=w_{i, A}^{n}
$$

if the correct permutation of the roots is used.

Conversely, let $x$ be a fixed point and, without loss of generality, let $\sigma$ be identity. Then $w_{i, A}^{n}=w_{i, A}^{n+1}=w_{i, A}$. This means that the roots of $P_{n}$ coincide with the roots of $P_{n+1}$. As both polynomials have the same roots and the same constant term, this shows that all coefficients are equal, which implies $a_{i, 1}^{A, n}+a_{i, 1}^{B, n}=a_{i, 1}$, and that equations $a_{i, 1}$ are satisfied. Consequently, $x$ solves the system.

Example 1 Let the molecule be described by $M=(G, W)$ with

$$
\begin{gathered}
G=\left(g_{1}, g_{2}, g_{3}, g_{4}, g_{A}, g_{B}\right)=\left(4 \times 10^{3}, 10,2 \times 10^{3}, 500,10^{3}, 10\right) \\
W=\left(w_{i, j}\right)_{i, j=1,2,3,4, A, B}=\left(\begin{array}{cccccc}
1 & 0.1 & 0.1 & 0.05 & 10 & 1 \\
0.1 & 1 & 0.5 & 0.5 & 10^{3} & 2 \times 10^{3} \\
0.1 & 0.5 & 1 & 0.05 & 10^{2} & 10 \\
0.05 & 0.5 & 0.05 & 1 & 10^{2} & 20 \\
10 & 10^{3} & 10^{2} & 10^{2} & 1 & 0.1 \\
1 & 2 \times 10^{3} & 10 & 20 & 0.1 & 1
\end{array}\right)
\end{gathered}
$$

For the sake of a clear view, we use a matrix notation for $W$ which repeats information but underlines which pairwise interaction is described. The binding polynomial of the 
molecule is given by

$$
\begin{aligned}
P_{M}(\Lambda, \kappa)= & 10^{22} \Lambda^{4} \kappa^{2}+2.5001 \times 10^{16} \Lambda^{4} \kappa+250000 \Lambda^{4} \\
& +5.1002 \times 10^{20} \Lambda^{3} \kappa^{2}+1.800602 \times 10^{15} \Lambda^{3} \kappa+1575000 \Lambda^{3} \\
& +3.0190 \times 10^{16} \Lambda^{2} \kappa^{2}+2.69328 \times 10^{12} \Lambda^{2} \kappa+966500 \Lambda^{2} \\
& +2.3040 \times 10^{10} \Lambda \kappa^{2}+3.0054 \times 10^{8} \Lambda \kappa+6510 \Lambda \\
& +1000 \kappa^{2}+1010 \kappa+1
\end{aligned}
$$

The binding to the individual sites is illustrated in Fig. 1.

We used the iterative approach described in Proposition 2 to calculate 24 different decoupled molecules, which correspond to the different permutations of the roots of $P_{2}$. The titration curves of the individual binding sites of two different decoupled molecules $N$ and $K$ are illustrated in Fig. 1. The binding constants of the decoupled molecules are always coinciding and given by (rounded):

$$
G^{D e}=(6358.026,150.328,1.468,0.178,1009.009,0.991) .
$$

The interaction constants of two chosen decoupled molecules $N, K$ are (rounded)

$$
\begin{aligned}
W_{N}= & \left(\begin{array}{cccccc}
1 & 1 & 1 & 1 & 45.323 & 75.119 \\
1 & 1 & 1 & 1 & 56.358 & 162.473 \\
1 & 1 & 1 & 1 & 487.352 & 23.900 \\
1 & 1 & 1 & 1 & 79.618 & 1.384 \\
45.323 & 56.358 & 487.352 & 79.618 & 1 & 1 \\
75.119 & 162.473 & 23.900 & 1.384 & 1 & 1
\end{array}\right) \\
W_{K}= & \left(\begin{array}{cccccc}
1 & 1 & 1 & 1 & 45.336 & 6.810 \times 10^{-05} \\
1 & 1 & 1 & 1 & 56.464 & 2550.214 \\
1 & 1 & 1 & 1 & 486.320 & 23.950 \\
1 & 1 & 1 & 1 & 79.613 & 97028.384 \\
45.336 & 56.464 & 486.320 & 79.613 & 1 & 1 \\
6.810 \times 10^{-5} & 2550.214 & 23.950 & 97028.384 & 1 & 1
\end{array}\right)
\end{aligned}
$$

Remark 1 (a) The fixed point algorithm described in Proposition 2 can easily be generalized to a situation of $(n, 2)$ binding sites. Only the degree of the polynomial whose roots have to be calculated increases.

(b) We note that it is not clear whether this procedure will always be attracted by its fixed point. However, our numerical test suggest that it converges quickly.

(c) The algorithm described in Proposition 2 can also be used with site $B$ as reference site $\left(a_{i, 1}^{B, 0}:=a_{i, 1}\right.$, etc $\left.\ldots\right)$. In all examples we calculated, this altered procedure led to another molecule with different sites $A$ and $B$. However, the titration curves of sites $1, \ldots, 4$ only depend on the chosen permutation of the products $w_{i, A} w_{i, B}$, and not on the choice of the reference site. In particular, this shows that our iterative approach has at least two (which equals $n_{2}$ !) fixed points for any permutation of the products $\left(w_{i, A} w_{i, B}\right)_{i=1, \ldots, 4}$. Switching the reference 


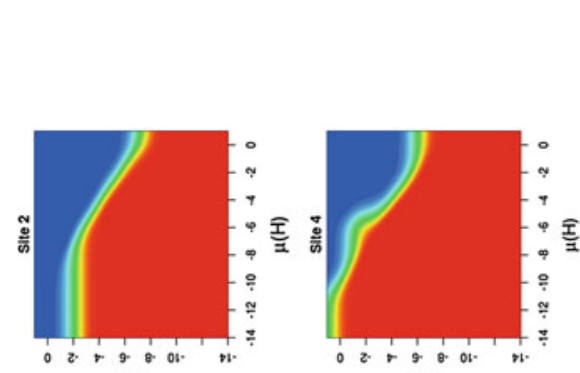

(อ) 1

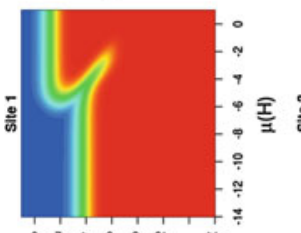

(อ) 1

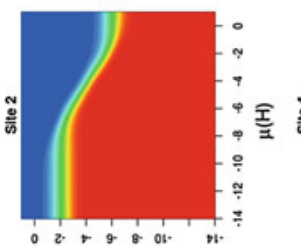

(อ) 1

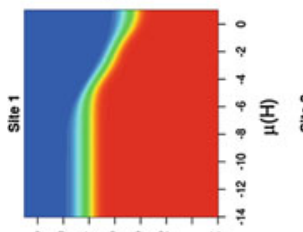

(ə) 1

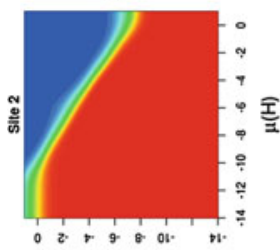

(ə) $\mathrm{r}$

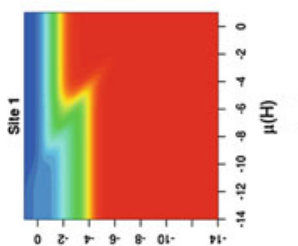

(ə)ท1 (ә) $\mathrm{r}$

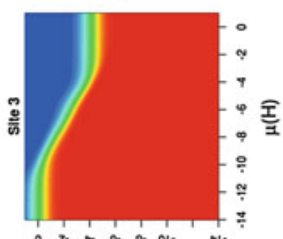

(ә) $\mathrm{r}$

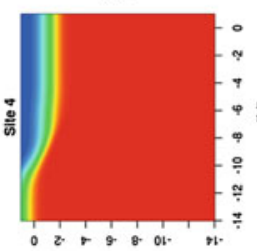

(ə) $\mathrm{r}$

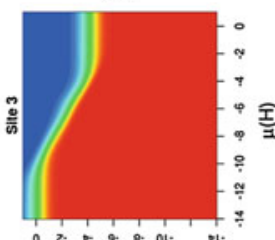

(ə)ri

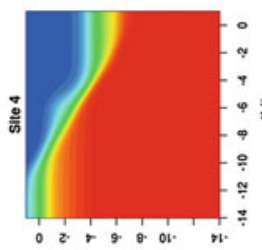

(ə)r

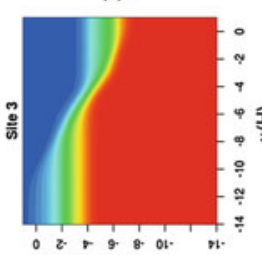

(ә) 11

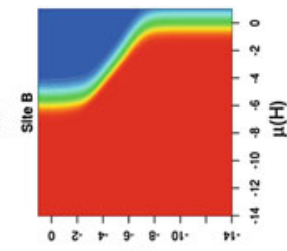

(ə) $\mathrm{rl}$

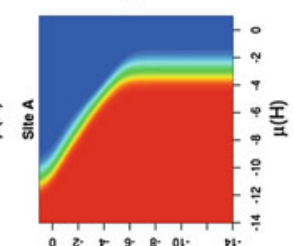

(ə) $\mathrm{ri}$

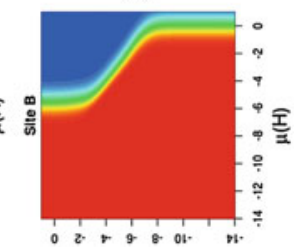

(ə) 1

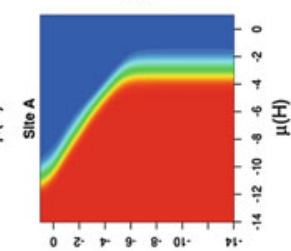

(ə) $\mathrm{r}$

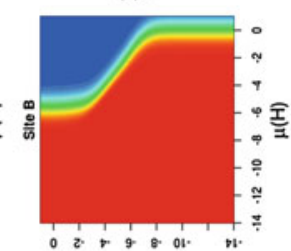

(e)r

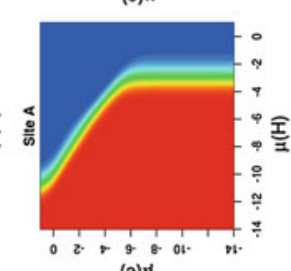

(ə) 11

홍ㅎㅇ

5.

$\checkmark$ 능

踏

亏气

今

ชั ฮัँ

o.

i

部

의

结要

2 .

을

造

농

할 $11 . \bar{g}$

言

\&ु응

을 낭

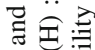

క 3

응

응

它

छ

क्षे

-

을 즐 跑

올

正

हo 은

$5 \frac{8}{0} \bar{\Xi}$

웡

$\Rightarrow$ 政

을.

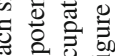

कृ

․ำ

잉ํㅇ

든

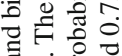

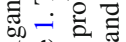

政

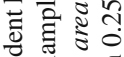

혼

웡ㄴㅇㅇ

จิ

政

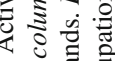

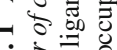

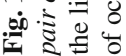




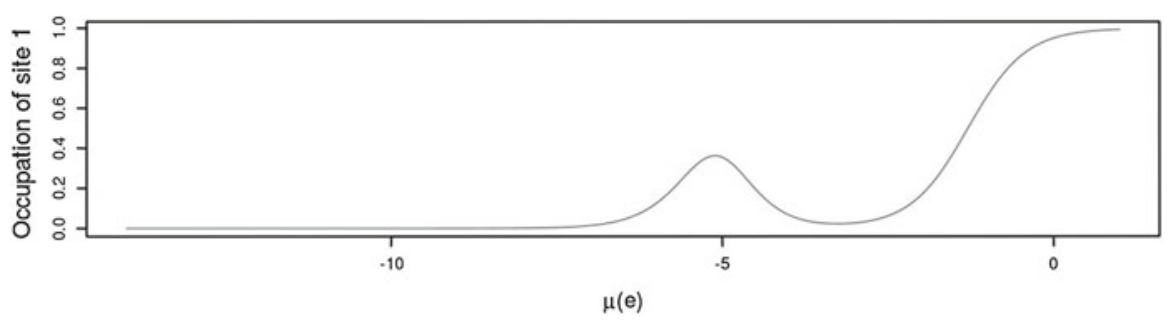

Fig. 2 Ligand binding to site 1 of the decoupled molecule $K$ of Example 1, dependent on the electron activity in chemical potential $\mu(\mathrm{e})$ for fixed $\mathrm{pH}=3=-\mu(\mathrm{H})$

site and calculating the decoupled molecules with our iterative procedure and all possible permutations of the roots gives additional 24 decoupled molecules sharing the same titration curves for protons. Moreover, not only the reference site, but also the starting point can be changed (e.g. $a_{i, 1}^{A, 0}:=\frac{1}{2} a_{i, 1}$ ). Yet, we do not know how the choice of the starting point and reference site determines which fixed point will be reached. It might be the case that the choice of the reference site implies that a certain fixed point is attractive and the other one repulsive.

(d) Our implementation is based on the R function "polyroot" [11] and we regarded the permutation which was returned by this function as $i d \in \mathscr{S}_{4}$.

(e) Due to our numerical results we conjecture that the maximal number of decoupled systems of a molecule $M$ with $\left(n_{1}, n_{2}\right)$ sites is $n_{1} ! n_{2} !$.

Regarding the titration curve of site 1 of the decoupled molecule $K$ at $p H=3$ (Fig. 2) we can see an extreme form of secondary interaction, which we have already described in the first part of this work [7]: Even though none of the electron binding sites interact directly, for fixed $\mathrm{pH}$ value, their 1-dimensional titration curves are not of classical Henderson-Hasselbalch shape. Secondary interaction between the electron binding sites is a result of the interaction with the protons: As site 1 of the decoupled molecules has a high binding constant (compared to the other binding sites), it will be occupied at a comparatively low activity. With an increase of electron activity, more electrons will bind to the other sites which will enhance the binding of the protons, in particular to site B. However, this decreases the affinity of electrons to site 1, due to the small interaction constant $w_{1, B}$ of molecule $K$.

Analogously to our observation in [7] we see here once more that for decoupled molecules the area of transition from 0.1 to 0.9 occupation probability is small, compared to the original molecule (sites 1,3 ).

\section{Unique features shared by all decoupled molecules}

Fig. 1 creates the suspicion that the binding curves of the individual sites for ligand one of the decoupled system $N$ in Example 1 are similar to those of the decoupled molecule $K$ in a certain way: The titration curve of site 1 of molecule $K$ seems to share the "right part" with site 4 of molecule $N$ and its "left part" seems to be identical to the "left part" of site 1 of molecule $N$. We have described this observation 
already for the case of $\left(n_{1}, 1\right)$ binding sites [7]. Analogously, to the first part of this work we want to identify unique features all decoupled molecules share. We will have a look on the microstate constants of the different molecules first. Table 1 lists the non-trivial microstate constants of the molecules $M, N$ and $K$ of Example 1. Microstates constants which are not listed are identical for the molecules $N$ and $K$ (when the permutation of the binding constants is fixed). We see here, that the microstate constants of macrostate $(1,1)$ of molecules $N$ and $K$ are not permutations of each other. However, for all macrostates in which the binding sites for one type of ligand are fully occupied, the corresponding microstate constants are permutations. We can prove this statement in general.

\section{Proposition 3 Let}

$$
M=\left(g_{1}^{M}, \ldots, g_{n_{1}}^{M}, g_{A_{1}}^{M}, \ldots, g_{A_{n_{2}}}^{M}, w_{1,2}^{M}, \ldots, w_{A_{n_{2}-1}, A_{n_{2}}}^{M}\right)
$$

be a molecule with $n_{1}$ binding sites for ligand $L_{1}$ and $n_{2}$ binding sites for ligand $L_{2}$. Moreover, let the order of the sites in the decoupled molecules be fixed to the same permutations. Then the following statements hold:

(a) For any microstate $k$ with only one type of ligand bound, all decoupled molecules share the same microstate constant $g(k)$.

(b) For every macrostate $\left(i, n_{2}\right)$, there exist $\left(\begin{array}{c}n_{1} \\ i\end{array}\right)$ numbers such that for any decoupled molecule the tuple of its constants of microstates belonging to this macrostate is a permutation of these numbers. Analogously, this statements holds for macrostates $\left(n_{1}, j\right)$.

(c) The permutation of microstate constants of macrostate $\left(1, n_{2}\right)$ fixes the permutations of the microstate constants of all other macrostates $\left(i, n_{2}\right)$. Analogously, for macrostate $\left(n_{1}, 1\right)$ and $\left(n_{1}, j\right)$.

Proof (a) As the permutation of the binding sites is fixed, and since the constants $g(k)$ are the product of the binding constants they are identical for all decoupled molecules.

(b) Let $k$ be a microstate of macrostate $\left(1, n_{2}\right)$. Its constant is given by

$$
g(k)=g_{i} g_{A_{1}} g_{A_{2}} \ldots g_{A_{n_{2}}} w_{i, A_{1}} \ldots w_{i, A_{n}} .
$$

The coefficients $a_{n_{1}, n_{2}}, a_{n_{1}-1, n_{2}}, \ldots, a_{1, n_{2}}$ are enough to calculate these constants which correspond to the roots of a polynomial. Thus, for any decoupled molecule the constants correspond to a permutation of these roots as the decoupled molecule has to fulfill the equations given by $a_{n_{1}, n_{2}}, a_{n_{1}-1, n_{2}}, \ldots, a_{1, n_{2}}$ in particular. Let $k_{1}$ be a microstate of macrostate $\left(i, n_{2}\right)$. Then its constant is the product of $i$ microstate constants belonging to macrostate $\left(1, n_{2}\right)$ divided by

$$
\left(\prod_{j=1}^{n_{2}} g_{A_{j}}\right)^{i-1}
$$


Table 1 Microstate constants of molecules $M$ and the two corresponding decoupled molecules $N$ and $K$ of Example 1

\begin{tabular}{|c|c|c|c|c|}
\hline Macrostate & Microstate & $M$ & $N$ & $K$ \\
\hline \multirow[t]{8}{*}{$(1,1)$} & $(1,0,0,0,1,0)$ & $4 \times 10^{7}$ & $290,757,860$ & $290,757,860$ \\
\hline & $(0,1,0,0,1,0)$ & $10^{7}$ & $8,548,419$ & $8,564,541$ \\
\hline & $(0,0,1,0,1,0)$ & $2 \times 10^{8}$ & $721,821.7$ & $720,293.5$ \\
\hline & $(0,0,0,1,1,0)$ & $5 \times 10^{7}$ & $14,315.07$ & $14,314.21$ \\
\hline & $(1,0,0,0,0,1)$ & $4 \times 10^{4}$ & $473,343.6$ & 0.4291216 \\
\hline & $(0,1,0,0,0,1)$ & $2 \times 10^{5}$ & $24,206.05$ & $379,944.48$ \\
\hline & $(0,0,1,0,0,1)$ & $2 \times 10^{5}$ & 34.76863 & 34.84239 \\
\hline & $(0,0,0,1,0,1)$ & $10^{5}$ & 0.2443494 & $17,135.27$ \\
\hline \multirow[t]{12}{*}{$(2,1)$} & $(1,1,0,0,1,0)$ & $4 \times 10^{10}$ & $2.463328 \times 10^{12}$ & $2.468703 \times 10^{12}$ \\
\hline & $(1,0,1,0,1,0)$ & $8 \times 10^{11}$ & $208,001,453,616$ & $207,622,407,458$ \\
\hline & $(1,0,0,1,1,0)$ & $10^{11}$ & $4,125,056,936$ & $4,126,026,921$ \\
\hline & $(0,1,1,0,1,0)$ & $10^{12}$ & $6.115341 \times 10^{9}$ & $6.113904 \times 10^{9}$ \\
\hline & $(0,1,0,1,1,0)$ & $2.5 \times 10^{11}$ & $121,278,630$ & $121,500,042$ \\
\hline & $(0,0,1,1,1,0)$ & $5 \times 10^{11}$ & $10,240,671$ & $10,218,376$ \\
\hline & $(1,1,0,0,0,1)$ & $8 \times 10^{7}$ & $1.156100 \times 10^{10}$ & $1.645112 \times 10^{5}$ \\
\hline & $(1,0,1,0,0,1)$ & $8 \times 10^{7}$ & $1.660577 \times 10^{7}$ & 15.08632 \\
\hline & $(1,0,0,1,0,1)$ & $2 \times 10^{7}$ & $116,703.229$ & $7,419.357$ \\
\hline & $(0,1,1,0,0,1)$ & $2 \times 10^{9}$ & $849,193.2$ & $13,357,436.4$ \\
\hline & $(0,1,0,1,0,1)$ & $10^{9}$ & $5.968020 \times 10^{3}$ & $6.569103 \times 10^{9}$ \\
\hline & $(0,0,1,1,0,1)$ & $10^{8}$ & 8.572232 & $6.024124 \times 10^{5}$ \\
\hline \multirow[t]{8}{*}{$(3,1)$} & $(1,1,1,0,1,0)$ & $4 \times 10^{14}$ & $1.762208 \times 10^{15}$ & $1.762314 \times 10^{15}$ \\
\hline & $(1,1,0,1,1,0)$ & $5 \times 10^{13}$ & $3.494787 \times 10^{13}$ & $3.502202 \times 10^{13}$ \\
\hline & $(1,0,1,1,1,0)$ & $10^{14}$ & $2.950970 \times 10^{12}$ & $2.945415 \times 10^{12}$ \\
\hline & $(0,1,1,1,1,0)$ & $1.25 \times 10^{15}$ & $8.675993 \times 10^{10}$ & $8.673431 \times 10^{10}$ \\
\hline & $(1,1,1,0,0,1)$ & $8 \times 10^{10}$ & $405,581,389,998$ & $5,783,603$ \\
\hline & $(1,1,0,1,0,1)$ & $2 \times 10^{10}$ & $2,850,373,606$ & $2,844,339,211$ \\
\hline & $(1,0,1,1,0,1)$ & $2 \times 10^{9}$ & $4,094,166$ & 260,837 \\
\hline & $(0,1,1,1,0,1)$ & $5 \times 10^{11}$ & $2.093692 \times 10^{5}$ & $2.309452 \times 10^{11}$ \\
\hline \multirow[t]{2}{*}{$(4,1)$} & $(1,1,1,1,1,0)$ & $2.5 \times 10^{16}$ & $2.50009 \times 10^{16}$ & $2.50009 \times 10^{16}$ \\
\hline & $(1,1,1,1,0,1)$ & $10^{12}$ & $9.99964 \times 10^{10}$ & $9.99964 \times 10^{10}$ \\
\hline \multirow[t]{4}{*}{$(1,2)$} & $(1,0,0,0,1,1)$ & $4 \times 10^{7}$ & $2.164640 \times 10^{10}$ & $1.962988 \times 10^{4}$ \\
\hline & $(0,1,0,0,1,1)$ & $2 \times 10^{10}$ & $1.376484 \times 10^{9}$ & $2.164640 \times 10^{10}$ \\
\hline & $(0,0,1,0,1,1)$ & $2 \times 10^{9}$ & $17,097,219$ & $17,097,219$ \\
\hline & $(0,0,0,1,1,1)$ & $10^{9}$ & $1.962988 \times 10^{4}$ & $1.376484 \times 10^{9}$ \\
\hline
\end{tabular}


Table 1 continued

\begin{tabular}{lllll}
\hline Macrostate & Microstate & $M$ & $N$ & $K$ \\
\hline$(2,2)$ & $(1,1,0,0,1,1)$ & $8 \times 10^{13}$ & $2.979592 \times 10^{16}$ & $4.249163 \times 10^{11}$ \\
& $(1,0,1,0,1,1)$ & $8 \times 10^{12}$ & $3.700932 \times 10^{14}$ & $3.356164 \times 10^{8}$ \\
& $(1,0,0,1,1,1)$ & $2 \times 10^{12}$ & $4.249163 \times 10^{11}$ & $2.702022 \times 10^{10}$ \\
& $(0,1,1,0,1,1)$ & $2 \times 10^{16}$ & $2.353405 \times 10^{13}$ & $3.700932 \times 10^{14}$ \\
& $(0,1,0,1,1,1)$ & $10^{16}$ & $2.702022 \times 10^{10}$ & $2.979592 \times 10^{16}$ \\
& $(0,0,1,1,1,1)$ & $10^{14}$ & $3.356164 \times 10^{8}$ & $2.353405 \times 10^{13}$ \\
$(3,2)$ & $(1,1,1,0,1,1)$ & $8 \times 10^{18}$ & $5.094274 \times 10^{20}$ & $7.264887 \times 10^{15}$ \\
& $(1,1,0,1,1,1)$ & $2 \times 10^{18}$ & $5.848904 \times 10^{17}$ & $5.848904 \times 10^{17}$ \\
& $(1,0,1,1,1,1)$ & $2 \times 10^{16}$ & $7.264887 \times 10^{15}$ & $4.619706 \times 10^{14}$ \\
& $(0,1,1,1,1,1)$ & $5 \times 10^{20}$ & $4.619706 \times 10^{14}$ & $5.094274 \times 10^{20}$ \\
\hline
\end{tabular}

The constants of microstates which are not listed are identical for molecules $N$ and $K$

This proves $b$ ) and $c$ ).

Remark 2 We have already conjectured that the maximal number of decoupled molecules is $n_{1} ! n_{2}$ !. This number corresponds to the different permutations of the microstate constants of the macrostates $\left(1, n_{2}\right)$ and $\left(n_{1}, 1\right)$. However, to prove our conjecture we would have to show, that for a fixed choice of these microstate constants, the remaining equations have a unique simultaneous solution. This equals proving the DSR for two types of ligands generally.

\section{Decoupling a molecule with $(3,3)$ binding sites}

Finally, we show how the algorithm of Proposition 2 can be extended to more than two binding sites for both ligands by presenting an iterative procedure for the case of $(3,3)$. Let

$$
\begin{aligned}
P_{M}(\Lambda, \kappa)= & a_{3,3} \Lambda^{3} \kappa^{3}+a_{3,2} \Lambda^{3} \kappa^{2}+a_{3,1} \Lambda^{3} \kappa+a_{3,0} \Lambda^{3} \\
& +a_{2,3} \Lambda^{2} \kappa^{3}+a_{2,2} \Lambda^{2} \kappa^{2}+a_{2,1} \Lambda^{2} \kappa+a_{2,0} \Lambda^{2} \\
& +a_{1,3} \Lambda \kappa^{3}+a_{1,2} \Lambda \kappa^{2}+a_{1,1} \Lambda \kappa+a_{1,0} \Lambda \\
& +a_{0,3} \kappa^{3}+a_{0,2} \kappa^{2}+a_{0,1} \kappa+1
\end{aligned}
$$

be a binding polynomial. We look for a corresponding decoupled molecule $N$. Let the sites for ligand $L_{1}$ be denoted by $1,2,3$ and for ligand $L_{2}$ by $A, B, C$. The coefficients $\left(a_{i, 0}\right)_{i=1,2,3}$ and $\left(a_{0, j}\right)_{j=A, B, C}$ give the binding constants. Let a permutation be chosen, that is, the order of the sites be fixed. Then the roots of the polynomial

$$
P_{1}(x)=\frac{a_{3,3}}{g_{A} g_{B} g_{C}} x^{3}+\frac{a_{2,3}}{g_{A} g_{B} g_{C}} x^{2}+\frac{a_{1,3}}{g_{A} g_{B} g_{C}} x+1
$$


give the products $\left(g_{i} w_{i, A} w_{i, B} w_{i, C}\right)_{i=1,2,3}$. Analogously to the case of $(4,2)$ binding sites the choice of the permutation is an important step to distinguish between different solutions. Having solved this subsystem, system (12) is left to be solved. We use analogous substitutions to the case of $(4,2)$ binding sites to facilitate understanding the structure of the system $\left(a_{1,2}^{A B}\right.$ denotes the part of coefficient $a_{1,2}$ derived from microstates with sites $A$ and $B$ occupied):

$$
\begin{aligned}
\xi_{i}^{j k}:= & g_{i} w_{i, j} w_{i, k} \\
a_{3,2}^{j k}:= & g_{j} g_{k} \xi_{1}^{j k} \xi_{2}^{j k} \xi_{3}^{j k} \\
a_{2,2}^{j k}:= & g_{j} g_{k}\left(\xi_{1}^{j k} \xi_{2}^{j k}+\xi_{1}^{j k} \xi_{3}^{j k}+\xi_{2}^{j k} \xi_{3}^{j k}\right) \\
a_{1,2}^{j k}:= & g_{j} g_{k}\left(\xi_{1}^{j k}+\xi_{2}^{j k}+\xi_{3}^{j k}\right) \\
a_{3,1}^{j}:= & g_{j} g_{1} w_{1, j} g_{2} w_{2, j} g_{3} w_{3, j} \\
a_{2,1}^{j}:= & g_{j} g_{1} w_{1, j} g_{2} w_{2, j}+g_{j} g_{1} w_{1, j} g_{3} w_{3, j}+g_{j} g_{2} w_{2, j} g_{3} w_{3, j} \\
a_{1,1}^{j}:= & g_{j} g_{1} w_{1, j}+g_{j} g_{2} w_{2, j}+g_{j} g_{3} w_{3, j} \\
& \quad \text { with } i \in\{1,2,3\} \text { and } j, k \in\{A, B, C\}, j \neq k
\end{aligned}
$$

Thus, the systems consisting of equations $a_{3,2}^{j k}, a_{2,2}^{j k}, a_{1,2}^{j k}$ and $a_{3,1}^{j}, a_{2,1}^{j}, a_{1,1}^{j}$ are of well known form and we see that the remaining equations given by the bp are the sum of the three systems:

$$
\begin{aligned}
& a_{3,2}=a_{3,2}^{A B}+a_{3,2}^{A C}+a_{3,2}^{B C} \\
& a_{2,2}=a_{2,2}^{A B}+a_{2,2}^{A C}+a_{2,2}^{B C} \\
& a_{1,2}=a_{1,2}^{A B}+a_{1,2}^{A C}+a_{1,2}^{B C} \\
& a_{3,1}=a_{3,1}^{A}+a_{3,1}^{B}+a_{3,1}^{C} \\
& a_{2,1}=a_{2,1}^{A}+a_{2,1}^{B}+a_{2,1}^{C} \\
& a_{1,1}=a_{1,1}^{A}+a_{1,1}^{B}+a_{1,1}^{C}
\end{aligned}
$$

To solve this system of equations we used the iterative procedure described in Proposition 4 which is an extension of the algorithm of Proposition 2. 
Proposition 4 Let the algebraic system (12) be given. Moreover, let $\left(g_{i}\right)_{i=1, \ldots, 3, A, \ldots, C}$ and $\left(w_{i, A} w_{i, B} w_{i, C}\right)_{i=1, \ldots, 3}$ be known (fixed permutations are chosen). We consider the following algorithm:

$$
\begin{gathered}
a_{i, 2}^{A B, 0}:=a_{i, 2}, \quad i \in\{1,2,3\} \\
P_{n}(x):=\frac{a_{3,2}^{A B, n}}{g_{A} g_{B}} x^{3}+\frac{a_{2,2}^{A B, n}}{g_{A} g_{B}} x^{2}+\frac{a_{1,2}^{A B, n}}{g_{A} g_{B}} x+1 \\
\left(z_{1}^{n}, z_{2}^{n}, z_{3}^{n}\right):=\text { the roots of } P_{n} \\
w_{i, A}^{n} w_{i, B}^{n}:=-\frac{1}{z_{\sigma^{n}(i)} g_{i}} \\
w_{i, C}^{n}:=\frac{w_{i, A} w_{i, B} w_{i, C}}{w_{i, A}^{n} w_{i, B}^{n}}
\end{gathered}
$$

Calculate $a_{i, 1}^{C, n}$ using equations $\left\{a_{i, 1}^{C}\right\}_{i=1,2,3}$ of (11) and $w_{i, C}^{n}$.

Use the procedure of Proposition 2 with a sequence of permutations $\sigma_{2}^{n}$ and the condition on the products $w_{i, A}^{n} w_{i, B}^{n}$ to calculate $w_{i, A}^{n}$ and $w_{i, B}^{n}$

$$
\text { from } a_{i, 1}-a_{i, 1}^{C, n}=a_{i, 1}^{A, n}+a_{i, 1}^{B, n} \text {. }
$$

$$
\text { Use } w_{i, A}^{n}, w_{i, B}^{n}, w_{i, C}^{n} \text { to calculate } a_{i, 2}^{A C, n} \text { and } a_{i, 2}^{B C, n} \text {. }
$$

$$
\text { Restart with } a_{i, 2}^{A B, n+1}:=a_{i, 2}-a_{i, 2}^{A C, n}-a_{i, 2}^{B C, n} \text {. }
$$

Then $x=\left(w_{1, A}, w_{1, B}, w_{1, C}, w_{2, A}, w_{2, B}, w_{2, C}, w_{3, A}, w_{3, B}, w_{3, C}\right)$ is a solution to system (12) which satisfies the conditions on the products $\left(w_{i, A} w_{i, B} w_{i, C}\right)_{i=1, \ldots, 3}$ if and only if there exist permutations $\sigma_{1}, \sigma_{2} \in \mathscr{S}_{3}$ such that $x$ is a fixed point of the algorithm with $\sigma^{n}=\sigma_{1}$ and $\sigma_{2}^{n}=\sigma_{2}$.

Proof Let $x=\left(w_{1, A}, w_{1, B}, w_{1, C}, w_{2, A}, w_{2, B}, w_{2, C}, w_{3, A}, w_{3, B}, w_{3, C}\right)$ be a solution to system (12) which satisfies the conditions on the products $\left(w_{i, A} w_{i, B} w_{i, C}\right)_{i=1, \ldots, 3}$.

Let $w_{i, A}^{n}=w_{i, A}, w_{i, B}^{n}=w_{i, B}$ and $w_{i, C}^{n}=w_{i, C}$. As $x$ solves the system, $a_{i, 2}^{A C, n}=$ $a_{i, 2}^{A C}$ and $a_{i, 2}^{B C, n}=a_{i, 2}^{B C}$ and consequently $a_{i, 2}^{A B, n+1}=a_{i, 2}^{A B}$. The roots of the polynomial give exact solutions $w_{i, A}^{n+1} w_{i, B}^{n+1}$ and thus exact solutions $w_{i, C}^{n+1}=w_{i, C}$, if the appropriate permutation $\sigma_{1}$ is used. This means $a_{i, 1}^{C, n+1}=a_{i, 1}^{C}$. As $w_{i, A}^{n+1} w_{i, B}^{n+1}=w_{i, A} w_{i, B}$, if an appropriate permutation $\sigma_{2}$ is used in the procedure of Proposition 2, $w_{i, A}$ and $w_{i, B}$ will be fixed. Consequently,

$$
a_{i, 2}^{A B, n+1}=a_{i, 2}^{A B, n}=a_{i, 2}^{A B} \text { and } P_{n}=P_{n+1}
$$

Conversely, let $x=\left(w_{1, A}, w_{1, B}, w_{1, C}, w_{2, A}, w_{2, B}, w_{2, C}, w_{3, A}, w_{3, B}, w_{3, C}\right)$ be a fixed point and $\sigma_{1}, \sigma_{2}$ be identity (without loss of generality). Then: 
$w_{i, A}^{n} w_{i, B}^{n}=w_{i, A}^{n+1} w_{i, B}^{n+1} \Rightarrow P_{n}=P_{n+1} \Rightarrow a_{i, 2}^{A B, n}=a_{i, 2}^{A B, n+1}$. This means $x$ satisfies all equations given by $a_{i, 2}$. Since $x$ is a fixed point $\left(w_{i, A}, w_{i, B}\right)_{i=1,2,3}$ has to be a fixed point of the iterative procedure described in Proposition 2. Since $a_{i, 1}^{C, n}=a_{i, 1}^{C, n+1}$, this means $\left(w_{i, A}, w_{i, B}\right)_{i=1,2,3}$ also solve $a_{i, 1}^{A, n}+a_{i, 1}^{B, n}+a_{i, 1}^{C, n}=a_{i, 1}$ which shows that $x$ solves the system.

We implemented the iterative procedure described in Proposition 4 to give an example with $(3,3)$ binding sites.

Example 2 Let the molecule be described by $M=(G, W)$ with

$$
\begin{gathered}
G=\left(g_{1}, g_{2}, g_{3}, g_{A}, g_{B}, g_{C}\right)=\left(4 \times 10^{3}, 10,2 \times 10^{3}, 500,10^{3}, 10\right) \\
W=\left(w_{i, j}\right)_{i, j=1,2,3, A, B, C}=\left(\begin{array}{cccccc}
1 & 0.001 & 0.01 & 10 & 10 & 1000 \\
0.001 & 1 & 0.05 & 100 & 1000 & 2000 \\
0.01 & 0.05 & 1 & 100 & 100 & 1000 \\
10 & 100 & 100 & 1 & 0.001 & 0.01 \\
10 & 1000 & 100 & 0.001 & 1 & 0.05 \\
1000 & 2000 & 1000 & 0.01 & 0.05 & 1
\end{array}\right)
\end{gathered}
$$

Then two decoupled molecules are given by $N=\left(G_{d e}, W_{N}\right)$ and $K=\left(G_{d e}, W_{K}\right)$ with

$$
\begin{aligned}
& G_{d e}=\left(g_{1}, g_{2}, g_{3}, g_{A}, g_{B}, g_{C}\right)=(5996.485,13.51409,4.936015 \\
& \times 10^{4}, 1509.304,0.6932945,2.389161 \times 10^{-3} \text { ) } \\
& W_{N}=\left(\begin{array}{cccccc}
1 & 1 & 1 & 46.94515 & 514.7757 & 153.93242 \\
1 & 1 & 1 & 180.15859 & 395.1010 & 96.96765 \\
1 & 1 & 1 & 1645.26622 & 341.0639 & 13.88127 \\
46.94515 & 180.15859 & 1645.26622 & 1 & 1 & 1 \\
514.7757 & 395.1010 & 341.0639 & 1 & 1 & 1 \\
153.93242 & 96.9676 & 13.88127 & 1 & 1 & 1
\end{array}\right) \\
& W_{K}=\left(\begin{array}{cccccc}
1 & 1 & 1 & 46.94539 & 514.6135 & 2.654036 \times 10^{-5} \\
1 & 1 & 1 & 180.15843 & 395.1741 & 23184.93 \\
1 & 1 & 1 & 1645.25945 & 341.1083 & 336723.6 \\
46.94539 & 180.15843 & 1645.25945 & 1 & 1 & 1 \\
514.6135 & 395.1741 & 341.1083 & 1 & 1 & 1 \\
2.654036 \times 10^{-5} & 23184.93 & 336723.6 & 1 & 1 & 1
\end{array}\right)
\end{aligned}
$$

The titration curves of all individual sites of molecules $M, N, K$ are illustraed in Fig. 3. 


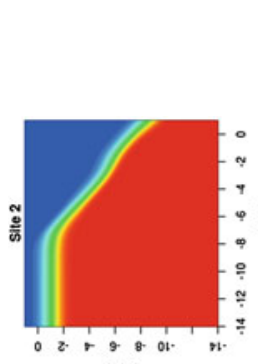

(ə)r

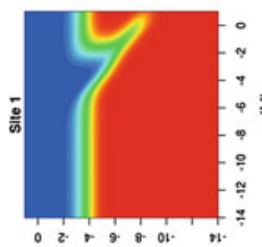

(ə) $\mathrm{H}$

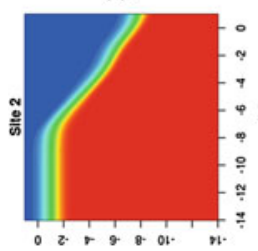

(ө)r1

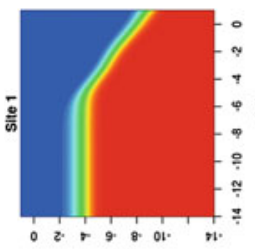

(ə)ri

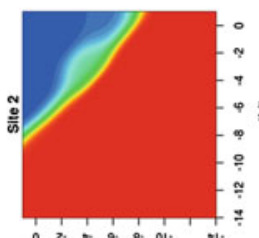

(ə) $\mathrm{r}$

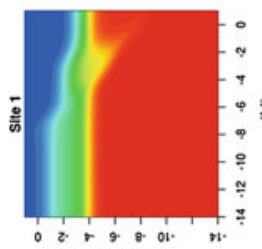

(ә) $\mathrm{r}$

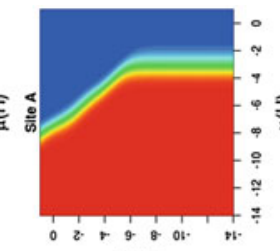

(ə) $\mathrm{r}$

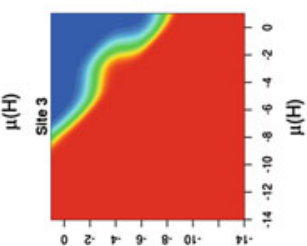

(อ) 1

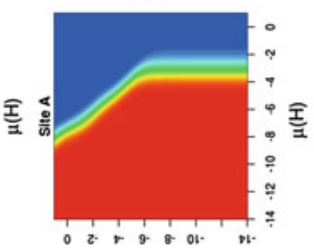

(ə) 11

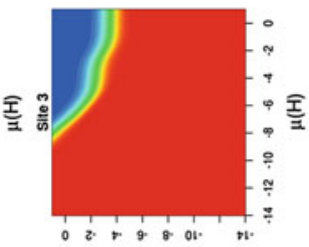

(ө) 11

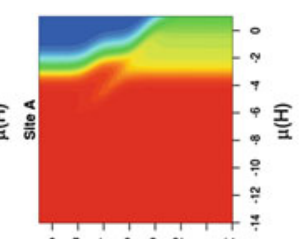

(ə) $\mathrm{r}$

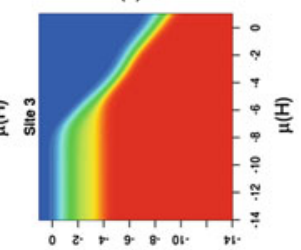

(ə) $n$

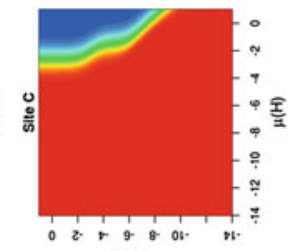

(ə) $\mathrm{r}$

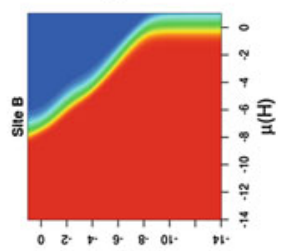

(ə))1

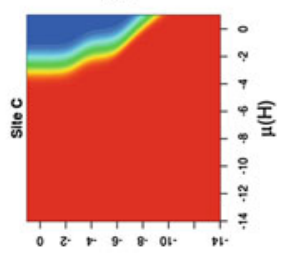

(ə))

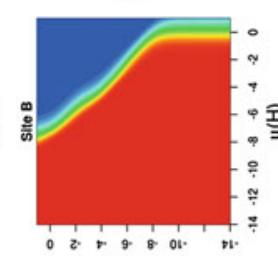

(ə) $\mathrm{r}$

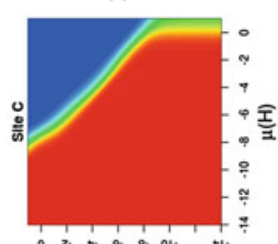

(ə) 1

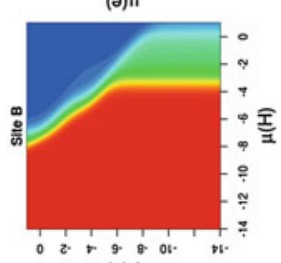

(ə) 1

홍ㅇㅇ

을

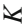

들

ฮ

ङ

ป $\frac{0}{3}$

00.

छ

¿

졿

इ

乙 రृ

\&

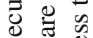

픙

일

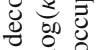

눙

을

इ

离 0

¿

पे

焉芯

ए)

$\sum$ 잉

을

웡

든

.્ّ

웜

늉 항

$\Xi$ 㽞

쁠

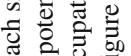

¿

일. 뜽 흥

哭寻

次

ป Еै

洁 을

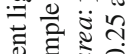

ฮิ

ปิ

하유

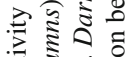

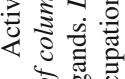

m

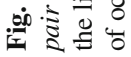




\section{Summary and outlook}

We investigated the titration curves of molecules binding more than one binding sites for two different ligands, and showed that all decoupled molecules share a certain set of microstate constants. Moreover, we presented numerical calculation methods to find decoupled molecules. Future work might investigate how decoupled molecules can highlight properties of the corresponding original molecule.

Acknowledgments We would like to thank Tobias Dorsch, Alexander Malinowski and Kristin Blumenröther for helpful discussions.

Open Access This article is distributed under the terms of the Creative Commons Attribution License which permits any use, distribution, and reproduction in any medium, provided the original author(s) and the source are credited.

\section{References}

1. G.K. Ackers, M.A. Shea, F.R. Smith, Free energy coupling within macromolecules: the chemical work of ligand binding at the individual sites in co-operative systems. J. Mol. Biol. 170, 223-242 (1983)

2. D. Bashford, M. Karplus, Multiple-site titration curves of proteins: an analysis of exact and approximate methods for their calculation. J. Phys. Chem. 95(23), 9556-9561 (1991)

3. E. Bombarda, G.M. Ullmann, pH-dependent pKa values in proteins-a theoretical analysis of protonation energies with practical consequences for enzymatic reactions. J. Phys. Chem. B 114(5), 1994-2003 (2010); PMID: 20088566

4. C.R. Cantor, P.R. Schimmel, Biophysical Chemistry. Part III. The Behavior of Biological Macromolecules, 1st edn. (W. H. Freeman, San Francisco, 1980)

5. K. Hasselbalch, Die Berechnung der Wasserstoffzahl des Blutes aus der freien und gebundenen Kohlensäure desselben, und die Sauerstoffbindung des Blutes als Funktion der Wasserstoffzahl (Julius Springer, Berlin, 1916)

6. L.J. Henderson, The Fitness of the Environment (Macmillan Company, New York, 1913)

7. J.W.R. Martini, M. Schlather, G.M. Ullmann, On the interaction of two different types of ligands binding to the same molecule part I: basics and the transfer of the decoupled sites representation to systems with $\mathrm{n}$ and one binding sites. J. Math. Chem. (2012). doi:10.1007/s10910-012-0107-5

8. J.W. Martini, G. Ullmann, A mathematical view on the decoupled sites representation. J. Math. Biol. 1-27 (2012). doi: 10.1007/s00285-012-0517-x

9. A. Onufriev, D.A. Case, G.M. Ullmann, A novel view of $\mathrm{pH}$ titration in biomolecules. Biochemistry 40(12), 3413-3419 (2001)

10. A. Onufriev, G.M. Ullmann, Decomposing complex cooperative ligand binding into simple components: connections between microscopic and macroscopic models. J. Phys. Chem. B 108(30), 1115711169 (2004)

11. R Core Team, R: A Language and Environment for Statistical Computing (R Foundation for Statistical Computing, Vienna, 2012). ISBN 3-900051-07-0

12. C. Tanford, J.G. Kirkwood, Theory of protein tiration curves. I. General equations for impenetrable spheres. J. Am. Chem. Soc. 79(20), 5333-5339 (1957) 